\section{Speculations on micromolecular evolution}

The Genetic Mechanism and the Origin of Life. By L. S. Dillon. Pp. 563. (Plenum: New York and London, 1978.)

I Found this to be a strange book. The list of almost 3,100 references occupies 127 of its 562 pages. This does not include the space in the text occupied by citations; I counted 23 on one page. Obviously, only perfunctory mention can be made of most articles to which the citations refer. An analogy to describe the organisation of the first five chapters is that it seemed as if the author had been out in the fields with a butterfiy net, industriously scooping up insects, which he sets before us in a display bewildering in its variety. His collection of topics includes everything from the origin of life, to the complete two-dimensional structure of MS-2 RNA, and the amino acid sequences of six calf-thymus and sea-urchin histones.

After this tour-de-force, the author proceeds to invent new theories from the stepwise evolutionary formulation of larger amino acids from smaller ones, such as alanine from glycine, and valine from aspartic acid. He says, for example, "In the author's laboratory, continuous bubbling of $\mathrm{CO}_{2}$ through a 0.1 [sic] aqueous solution of alanine at ambient and elevated temperatures for a week failed to produce any detectable aspartic acid (Dillon, unpublished)", but he doesn't give up; he next says, "Hence, the addition can probably be made only through enzymatic mediation". He does not mention that aspartic acid is formed biologically by transamination, but not from alanine. Grandiose but unrealistic schemes are also set forth for "chemical evolution" of lysine, isoleucine and other amino acids from aspartic acid.

In chapter 7, Dillon proceeds to take sequences of tRNAs apart into small pieces and put them together again. $\mathrm{He}$ says that the absence of unmodified adenosine from the first site in anticodons is included in constraints which "to the author's knowledge . . . have not been previously noted in the literature". Actually, this is a wellknown characteristic and has a familiar explanation, not mentioned by Dillon. Adenosine in the first position of anticodons is deaminated to inosine, (Biochim. Biophys. Acta, 213, 352, 1970). which wobble-pairs with three different bases. The incorrect statement is made on p296 that "the triplet -CAA is consistently present at the 3'terminus of the molecule" of tRNA.
The next chapter, on "tRNA evolution", atempts at great lengths to construct a phylogeny by comparing base sequences of various arms of tRNA molecules. Such a procedure is at variance with results obtained by aligning and comparing the complete sequences of tRNA molecules ( $J$. Molec. Biol. 78, 91; 1973), a method which shows that sequence changes have been so extensive as to obscure any phylogenetic relationship of nearly all tRNAs except for a few closely related pairs.

In chapter 9, Dillon goes butterflycollecting for odds and ends among the structures of viruses. At one point he briefiy notes, but does not describe, the "blocked complex 5'-terminal struc-

\section{Protein \\ phosphorylation}

Cyclic Nucleotides, Phosphorylated Proteins, and Neuronal Function. Distinguished Lecture Series of the Society of General Physiologists. Vol. 1. By P. Greengard. Pp. 124. (Raven: New York, 1978.) $\$ 15.60$.

IN this slim volume, Professor Greengard expounds his theory that protein phosphorylation is the final common pathway that mediates the biological effects of a wide variety of regulatory agents in addition to those that are known to act through cyclic AMP. It is really an extension of the 'second messenger' theory of cyclic AMP function, in that protein phosphorylation is depicted as the immediate and physiologically important result of increased cyclic AMP levels. However, Professor Greengard is nothing if not catholic in his enthusiasm for protein phosphorylation, and the actions of steroid and peptide hormones, insulin, interferon, all the neurotransmitters (excepting acetylcholine at nicotinic synapses) as well as the diverse effects of calcium ions are all envisaged by him as proceeding by this mechanism.

One's unease develops as the list increases; can the expression of all this biological diversity really be channelled through the simple phosphorylation of protein? It is clear that the regulation of glycolysis proceeds by cyclic AMPinduced protein phosphorylation. Other cases exist, but the argument from the particular to the general is not well founded. The mere correlation of varied cyclic nucleotides with protein phosphorylation in various circumstances is necessary but not sufficient to demonstrate it. It may be that protein phosphorylation is a side-effect of increased cyclic nucleotide levels rather than a mandatory stage in the expression of ture" in reovirus and polyhedrosis virus RNA, discovered by Miura et al. (p402). This is, of course, the "cap" of eukaryotic mRNA, an appendage which Dillon (p131) strangely ignored in describing the structure of eukaryotic mRNA. The book concludes (chapter 10) with highly imaginative (or imaginary) and grandiose scenarios for the origin of life, including schemes for the stepwise evolution of small viruses into large ones. The voluminous bibliography, following chapter 10 , is probably the most useful feature of the book.

Thomas H. Jukes

Thomas H. Jukes is in the Department of Biophysics and Medical Physics, University of California, Berkeley, California.

their regulatory activity.

And even if protein phosphorylation was a mandatory stage, does this tell us much about the processes anyway? After all even if the theory were true, the important questions surely are; what proteins in each individual case are phosphorylated and how does this affect their physiological activity? Professor Greengard would probably agree that these questions are important and protest that he is merely proposing a working hypothesis for the detailed investigation of individual cases. But the trouble is that protein phosphorylation as a working hypothesis is so general and vague that it does not really help us formulate specific questions about regulatory mechanisms.

I turn now to the particular role of protein phosphorylation in neuronal and synaptic activity, Professor Greengard's special interest. He has proposed a scheme whereby a neurotransmitter (presumably the author has noradrenaline in mind, though it is not specified) is bound to its postsynaptic receptor and activates adenyl cyclase; this stimulates protein phosphorylation which causes a permeability change and thus a change in the membrane potential. Now, as Professor Greengard shows, the phosphorylative and enzymic activities prescribed by the scheme are found in the synaptic region but it is by no means clear that phosphorylation of the membrane protein causes the membrane conductance change that is known to be caused by transmitter action. The best experimental evidence that the author can offer is that isoproterenol (known to cause an increase in cyclic AMP) causes in turkey erythrocytes an increase in sodium influx, and protein phosphorylation, the dose-and time-response curves of which are similar. There are various correlations but none distinguishes between protein phosphorylation being a result of an increased ion flux rather 